Chirurg 2014 · 85:641

DOI 10.1007/s00104-014-2793-z

Online publiziert: 18. Juni 2014

(c) Springer-Verlag Berlin Heidelberg 2014
O. Strobel · M.W. Büchler

Klinik für Allgemein-, Viszeral- und Transplantationschirurgie, Universität Heidelberg

Eine Phase-II-Studie

\section{Methoden}

\section{Originalpublikation}

D'Angelica MI, Correa-Gallego C, Paty PB et al (2014) Phase II trial of hepatic artery infusional and systemic chemotherapy for patients with unresectable hepatic metastases from colorectal cancer: conversion to resection and long-term outcomes. Ann Surg [Epub ahead of print] PMID: 24646562

\section{Hintergrund und Fragestellung}

Bei Patienten mit kolorektalen Lebermetastasen (KRLM) führen moderne systemische Chemotherapien zu einem medianen Überleben von etwa 20 Monaten; ein 5-Jahres-Überleben wird nur sehr selten beobachtet. Nach kompletter Resektion werden hingegen 5-Jahres-Überlebensraten von bis $\mathrm{zu} 50 \%$ beobachtet; bis $\mathrm{zu}$ 20\% der Patienten können als geheilt gelten. Allerdings haben die meisten Patienten nicht resektable KRLM. In retrospektiven Studien zur Resektion nach Downstaging primär nicht resektabler KRLM werden nach systemischer Chemotherapie Resektionsraten von $12-38 \%$ und nach kombinierter intraarterieller („hepatic-arterial infusion“, HAI) und systemischer Chemotherapie Resektionsraten von 47\% berichtet. Allerdings sind die Resektabilitätskriterien in diesen retrospektiven Studien oft nicht klar definiert.

D’Angelica et al. aus dem Memorial Sloan Kettering Cancer Center, einem ausgewiesenen Zentrum für Leberchirurgie, führten nun eine prospektive Phase-II-Studie zur HAI in Kombination mit systemischer Chemotherapie durch.

\title{
Kombinierte intraarterielle und systemische Chemotherapie bei nicht resektablen Lebermetastasen eines kolorektalen Karzinoms
}

Im Rahmen der prospektiven Phase-IIStudie wurden Patienten mit nicht resektablen KRLM ohne extrahepatische Tumormanifestation mit HAI (Floxuridine) in Kombination mit der besten verfügbaren systemischen Chemotherapie behandelt. Der primäre Endpunkt war die Resektabilitätsrate. Die Resektabilität wurde nach strengen Kriterien durch zwei hepatobiliäre Chirurgen und einen Radiologen festgelegt. Sekundäre Endpunkte waren die Ansprechrate (WHO-Kriterien), das Gesamtüberleben, das progressionsfreie Überleben und die Toxizität.

\section{Ergebnisse}

Zwischen 2007 und 2010 wurden $102 \mathrm{~Pa}$ tienten gescreent und 49 (48\%) eingeschlossen. Die meisten Patienten $(n=32$; $65 \%$ ) waren bereits systemisch vortherapiert und erhielten die Studienbehandlung als Zweit- ( $\mathrm{n}=21 ; 43 \%)$ oder Drittlinientherapie ( $n=11 ; 22 \%)$. Eine Grad-3/4-Toxizität wurde bei 20 (41\%) Patienten beobachtet. Patienten mit systemischer Bevacizumab-Therapie hatten häufig biliäre Komplikationen, weshalb Bevacizumab ab dem 25. Patienten nicht mehr eingesetzt wurde. Danach sank die Rate an Grad-3/4-Toxizität auf 28\%. Die Ansprechrate war 76\% ( $82 \%$ bei chemotherapienaiven und $72 \%$ bei systemisch vorbehandelten Patienten). Die Resektionsrate nach einem Median von 6 Monaten war 47\% (23/49 Patienten). Die Resektionen waren teils komplex und wurden bei 16 Patienten in Kombination mit lokal ablativen Verfahren durch- geführt. Es gab keine 90-Tage-Mortalität. Bei einem medianen Follow-up von 38 Monaten war das mediane Gesamtüberleben 38 Monate und das medianen progressionsfreie Überleben 13 Monate. Die Konversion zur Resektabilität war signifikant mit einem längeren Überleben assoziiert (3-Jahres-Überlebensrate $80 \%$ vs. $26 \%$ ). Bei der letzten Nachbeobachtung waren 10 der 49 Patienten (20\%) nach einem Median von 39 Monaten erkrankungsfrei.

\section{Diskussion und Fazit}

Die Autoren folgern, dass selektionierte Patienten mit streng definierten nicht resektablen Lebermetastasen durch eine Kombination aus HAl und systemischer Chemotherapie in einen resektablen $\mathrm{Zu}$ stand überführt werden können und von dieser Behandlung profitieren können. Diese Ergebnisse sind vielversprechend, insbesondere für Patienten mit Lebermetastasen, die nach systemischer Chemotherapie weiterhin nicht resektabel sind. Die Rolle einer zusätzlichen HAI im Vergleich zur systemischen Chemotherapie alleine sollte in randomisiert-kontrollierten Studien überprüft werden.

\section{Korrespondenzadresse}

\section{PD Dr. O. Strobel}

Klinik für Allgemein-, Viszeral- und Transplantationschirurgie, Universität Heidelberg, Im Neuenheimer Feld 110, 69120 Heidelberg Oliver.Strobel@med.uni-heidelberg.de

Interessenkonflikt. O. Strobel und M.W. Büchler geben an, dass kein Interessenkonflikt besteht. 